\title{
LA TECHNOLOGIE HABITÉE
}

La forme de vie globale des marchés financiers

Karin KNORR CETINA

Urs BRUEGGER

(C) VO: K. Knorr Cetina, U. Bruegger, "Inhabiting Technology: The Global Lifeform of Financial Markets", Current Sociology, May 2002, vol. 50(3): 389-405.

(C) 2002 by International Sociological Association.

(C) Réseaux n 122 - FT R\&D / Hermès Science Publications - 2003

Konstanzer Online-Publikations-System (KOPS)

URN: http://nbn-resolving.de/urn:nbn:de:bsz:352-opus-81234

URL: http://kops.ub.uni-konstanz.de/volltexte/2009/8123/ 

A quoi ressembleront les formes sociales globales du XXI siècle? Dans cet article, nous nous concentrons sur un modèle qui pourrait se généraliser. Il est né à la fin des années 1970 du monde des Etats-nations, un monde dont il s'est dégagé, mais sur l'existence duquel il compte (malgré sa puissance, cette forme de vie peut tout simplement disparaître dans certaines circonstances de régulation). Dans les années 1970, les Etats-Unis d'abord (1971), les grands pays européens (dont la Grande-Bretagne en 1979) ensuite et enfin le Japon au début des années 1980 ont aboli les contrôles dans le marché des changes ${ }^{1}$, mettant de fait un terme aux accords de Bretton Woods sur des taux de change fixes en vigueur depuis 1944 et permettant ainsi le commerce des devises à des fins spéculatives. En 1986, observent Hamilton et Biggart ${ }^{2}$, les salles de marché 3 semblaient en effervescence, avec une moyenne de 150 milliards de dollars américains et des pics de 250 milliards échangés à travers la planète, soit deux fois le volume de cinq ans auparavant. D'après la dernière étude triennale de la Banque des règlements internationaux (BIS), en avril 1998 le chiffre d'affaires quotidien moyen sur les instruments conventionnels d'opérations de change du monde entier était passé de 36,4 milliards de dollars en 1974 à 1,5 trillions de dollars ${ }^{4}$. Deux tiers de ce volume proviennent de transactions de gré à gré, c'est-à-dire de transactions bilatérales au sein d'un réseau mondial d'établissements financiers. Les banques avaient répondu rapidement aux opportunités commerciales

1. [NdT: ou marché des devises, Foreign Exchange en anglais].

2. HAMILTON, BIGGART, 1993.

3. [NdT: on désigne généralement par «salles de marché » (trading room ou dealing room) les départements des banques d'investissement, courtiers et autres établissements financiers au sein desquels se concentrent les activités de négociation sur les divers marchés financiers : marchés d'actions, marchés de produits obligataires, divers genres de marchés dérivés et marché des changes (les auteurs se concentrent dans cet article sur ce dernier). Le mot «trader» (ou «opérateur») désigne de manière générique tout employé d'établissement financier (travaillant habituellement en salle de marché) dont l'activité principale consiste à négocier sur les marchés financiers : intervenir en transmettant des ordres d'achat et de vente (pour le compte de clients ou de la banque qui l'emploie) et étudier puis mettre en œuvre des stratégies de profit].

4. BIS, 1998. 
survenues lors de la libéralisation des capitaux initiée par l'effondrement du système de Bretton Woods. Elles ont également répondu à une demande croissante stimulée par des taux de change et d'intérêt volatiles reflétant les diverses crises (par exemple, la crise énergétique de 1974) et à la croissance énorme des fonds de pension et autres formes d'investissement institutionnel.

Cette petite histoire de la croissance du marché des changes sert de toile de fond à une autre histoire, celle de l'émergence d'une forme de vie globale qui « habite la technologie» dans un sens fort littéral. Distribuée sur les trois principales zones de fuseaux horaires (Asie, Europe, Amérique) cette forme de vie est néanmoins centrée en et sur elle-même : c'est une forme de vie incarnée par ce marché et par ceux qui y participent. Dans cet article, nous développerons une analyse de ce que peut signifier pour un système technologique particulier le fait d'être «habité » par ses participants. Nous étudierons également comment ce réseau de négociation intra et interbancaire se constitue en «monde », en insistant sur le rôle des écrans, là où le réseau devient centré. L'un des objectifs majeurs de cet article est de contribuer à l'ouverture de la notion de réseau d'information et de communication en soulignant le rôle d'un tel centrage.

Le principe de base qui sous-tend notre argument est que les notions d'interaction et de réseau à l'usage dans maints domaines sociaux ignorent souvent la réalisation effective des réseaux : leur « réalisation » en tant que sphères centrées, relativement contraires à l'idée d'unités distanciées ou de nœuds connectés uniquement par des liaisons marchandes ou des relations sociales. Avant 1980, le marché des changes était effectivement structuré en termes de réseaux. Mais le marché des changes contemporain est mieux appréhendé en tant que plate-forme centrée et que structure de flux basée sur «l'apprésentation». La notion de technologie en tant qu'infrastructure externe d'échange d'informations masque le rôle constitutif d'un composant particulier de cette technologie: l'écran, avec tout le travail apprésentationnel des traders qu'il suppose et toute l'économie secondaire d'informations qu'il demande.

Nous étudierons dans un premier temps les concepts de réseau par rapport aux évolutions récentes du commerce et de la finance et nous examinerons la transition historique du marché des changes d'un marché en réseau à un marché en écran. Nous en viendrons ensuite à le caractériser en tant que 
«monde » et en tant que flux de ce marché. Nous nous appuyons sur les travaux d'Alfred Schutz pour expliciter en quoi ces caractéristiques diffèrent de celles de la réalité de la vie quotidienne qu'il a analysée, en soutenant que la temporalité, la contextualité et la localité spécifiques du «monde en écran » des traders sont liées à la constitution technologique de ce monde. Nous conclurons par une analyse de la transparence des marchés sur écran en tant que constitutive de leur matérialité informationnelle.

\section{MODÈLES DE RESEAUX}

Le concept de réseau est ancien en sociologie. On peut toutefois penser que son récent regain de popularité est ancré dans les changements rapides subis par une multitude de formes organisationnelles analysées par les sociologues. L'entreprise est l'une de ces formes: dans ce cas, le changement a été associé à un assouplissement de la forme organisationnelle bureaucratique qui était apparue dans le sillage de l'industrialisation. Dans la transformation culturelle à laquelle nous assistons, les entreprises adoptent une structure plus plate, s'appuient davantage sur le travail d'équipe et moins sur des hiérarchies explicites, se scindent en unités commerciales indépendantes et externalisent des activités - ce qui implique que les connexions horizontales au sein des et entre les entreprises non seulement complètent mais supplantent les regroupements et les connexions verticaux. Ces changements concernent autant les principes intra-entreprise de formation de groupes que la structure de gouvernance d'une société et les relations inter-entreprises.

La notion de réseau recouvre tous ces aspects. $\mathrm{Si}$, par le passé, les spécialistes des sciences sociales avaient une prédilection pour les concepts de bureaucratie et de hiérarchie, ils sont aujourd'hui plutôt férus des concepts de réseau et de connectivité. On peut ainsi se reporter aux travaux de Powell ${ }^{5}$ qui décrivent la progression de réseaux latéraux centrés sur les tâches au sein des entreprises et des réseaux de collaboration interentreprises dans le domaine des sociétés de biotechnologie, ainsi qu'aux travaux de Stark ${ }^{6}$ qui identifie et analyse les réseaux informels entre entreprises en Europe de l'Est, ou de Hamilton et Biggart, et de Gerlach ${ }^{7}$, qui décrivent

5. POWELL, KOPUT, SMITH-DOERR, 1996.

6. STARK, 1996.

7. HAMILTON, BIGGART, 1993 ; GERLACH, 1992. 
l'organisation et les changements sociaux au sein des entreprises japonaises, coréennes et chinoises en termes de différents types d'alliances et de réseaux, ou enfin de DiMaggio ${ }^{8}$ qui réunit diverses études dans cette tendance.

Cet engouement semble justifié à de nombreux égards. En tant que concept d'organisation, la notion de réseau repose en effet sur une solide convergence des changements organisationnels, des développements technologiques et des transformations culturelles plus générales qui soutiennent également la nature du concept de réseau en tant que modèle analytique, mais aussi en tant que publicité sur la façon dont le commerce devrait être pratiqué dans un domaine quelconque. Un des développements importants qui contribue au concept de réseau est certainement celui des technologies d'information et de communication, lesquelles reposent sur des liaisons électroniques entre régions géographiques et sont souvent décrites en termes de réseaux, toiles, circuits et nœuds. Celles-ci ont renforcé les tendances préexistantes vers des formes d'organisations en réseau et ont facilité certains de ces développements. Castells parle ainsi d'une société en réseau où «les flux de messages et d'images entre réseaux constituent le fil conducteur de base de notre structure sociale ${ }^{9} »$. Il conçoit les fonctions dominantes comme étant organisées en réseaux, tandis que des fonctions subordonnées se fragmentent et que, au niveau local, les personnes sont de plus en plus isolées et déconnectées les unes des autres. Si l'usine d'assemblage était l'exemple type de l'entreprise du $\mathrm{XX}^{\mathrm{e}}$ siècle, les technologies de l'information peuvent jouer un rôle similaire au $\mathrm{XXI}^{\mathrm{e}}$ siècle ; il paraît donc normal de se référer à tout dispositif institutionnel en termes de réseaux ${ }^{10}$.

Des facteurs culturels semblent également contribuer à l'essor de cette notion : une popularité mise en évidence par la préférence, croissante depuis les années 1980, des analystes à envisager les choses en termes de réseaux, même dans des domaines où les transitions soudaines vers des formes d'organisations en réseau ne sont pas évidentes. Cette tendance est illustrée par la théorie de l'acteur-réseau en sociologie des sciences et des techniques ${ }^{11}$. L'analyse des marchés, qui, galvanisée par Granovetter et sa

8. DIMAGGIO, 2001.

9. CASTELLS, 1996, p. 476-7.

10. DIMAGGIO, 2001, p. 39-40.

11. Par exemple : LAW, HASSARD, 1999. 
notion d'encastrement (embeddedness) de l'action économique dans les réseaux de relations ${ }^{12}$, est maintenant dominée par des approches « réseau », en est un autre exemple. La ligne de recherche dominante dans ce domaine se spécialise dans l'analyse des liens inter-organisationnels et relie, en fait, l'analyse organisationnelle et l'analyse des marchés en se concentrant sur l'étude de la nature des relations et des réseaux et l'incidence de ceux-ci sur le travail, la production, le crédit et l'investissement ${ }^{13}$. L'encastrement dans les réseaux est une notion théorique qui pose comme postulat l'interdépendance des réalités économiques avec la structure sociale. La recherche tente d'expliquer comment les activités économiques sont modelées par ce qui anime les relations dans lesquelles les acteurs sont engagés. C'est un postulat qu'il convient de distinguer de la théorie selon laquelle les entreprises se réorganisent elles-mêmes à la suite de transformations culturelles plus générales.

Néanmoins, malgré ces convergences le sentiment demeure qu'un concept aussi polyvalent que celui de «réseau » a également des limites majeures. En effet, les réseaux sont des structures sociales éparses et il est difficile de voir comment ils peuvent incorporer des formes d'interaction intense et dynamique, des composants symboliques et d'autres spécificités que nous observons dans des domaines concrets. En outre, les réseaux postulent et exigent de fait la distanciation entre les unités, les connexions venant combler cette distance. Dans l'imagerie du réseau, ce sont les connexions qui supportent la charge de la preuve dans l'explication d'une forme sociale. Mais l'accent mis sur les liens, leur nature et ce qui les anime, fait peu de cas des détails sur la façon dont les connexions sont implémentées. Ces détails comptent dans le formatage des processus d'organisation ou des formes sociales : il convient de savoir si c'est bien sur les liens existant dans un réseau (ou sur une autre structure) que repose une organisation donnée.

Sur les marchés analysés ici, les technologies d'information et de communication (médias transactionnels), fournissent non seulement des liens mais projettent également des intérêts, activités et événements locaux sur un espace symbolique commun : celui des écrans d'ordinateur. A l'image d'un réseau de prismes qui recueillent la lumière et la concentrent en un point, ces systèmes recueillent et concentrent les activités, les intérêts et les

12. GRANOVETTER, 1985.

13. Par exemple, BAKER, 1990 ; BAKER, FAULKNER, FISHER, 1998 ; DIMAGGIO, LOUCH, 1998 ; UZZI, 1999. 
événements à la surface des écrans d'ordinateur. Ces écrans eux-mêmes sont reproduits à l'identique dans toutes les salles de marché connectées, formant pour ainsi dire un énorme dispositif de miroitement composite. En termes plus phénoménologiques ${ }^{14}$, l'écran «apprésente» le marché : il amène l'invisible et le territorialement distant «près » des participants, ce qui le rend interactionnel et réactif.

\section{DES RÉSEAUX À L'ECRAN}

Il convient d'illustrer tout ceci par un cas concret, le marché des changes (ou marché des devises), que nous allons maintenant aborder. Examinons d'abord la salle de marché étudiée ${ }^{15}$. Environ deux cents traders (toutes activités confondues : actions, obligations et devises) travaillaient sur les salles de marché de la banque d'investissement internationale observée. Les traders des opérations interbancaires de change ne sont pas des courtiers qui négocient pour le compte de tiers, mais plutôt des teneurs de marché (market makers). Ils prennent leurs propres «positions » sur le marché en essayant de profiter des différences de cours en proposant des opérations aux autres participants du marché, conférant ainsi de la liquidité au marché et le soutenant, si nécessaire en réalisant des transactions allant contre leur propre position. L'ordre de grandeur des opérations de change réalisées via ces canaux est de plusieurs centaines de milliers de dollars par transaction et peut aller jusqu'à cent millions de dollars ou plus. A l'origine de ces transactions se trouvent des investisseurs institutionnels, des spéculateurs, des gestionnaires d'actifs, des banques centrales et autres acteurs qui veulent profiter des mouvements des cours des devises, ou qui ont besoin de devises pour s'engager ou se dégager d'investissements transnationaux (par exemple, dans le cas de fusions et acquisitions). Pour réaliser les transactions, les traders disposent de toute une palette de technologies: chaque opérateur a en face de lui jusqu'à cinq écrans qui affichent le marché et permettent d'y intervenir. L'écran est le moyen qui permet de conclure des transactions par le biais de contacts directs en temps réel, appelés

14. SCHUTZ, 1962, p. 294-305.

15. Cette étude est basée sur un travail ethnographique réalisé depuis 1997 dans une salle de marché d'une importante banque d'investissement internationale à Zurich et dans deux autres banques. Pour plus de détails, voir : KNORR CETINA, BRUEGGER, 2002a. 
« conversations ${ }^{16}$ », ou à travers l'Electronic Broker (ou système EBS), un système qui indique les offres d'achat et de vente et qui fait la liaison pour les transactions. Les écrans présentent le «noyau » du marché et la majeure partie de son contexte. Ils composent un monde autonome qui comporte « tout » ce dont il a besoin pour son existence et son maintien : au centre, les prix des transactions effectives et les conversations de négociation en cours ; dans un second cercle, les cours indicatifs, les informations sur les comptes et quelques nouvelles textuelles (en fonction de l'histoire actuelle du marché), ainsi que des nouvelles et commentaires supplémentaires constituant un troisième cercle d'informations.

Le marché n'a bien entendu pas toujours été sur écran. L'histoire du marché des changes depuis les années 1970 illustre la transition des réseaux vers un espace composite centralisé. Commençons par l'effondrement en 1971 des accords de Bretton Woods, qui avaient jusque-là fixé efficacement les taux de change. Avant cet effondrement, les marchés de change existaient également: les opérations de change étaient des échanges de devises transfrontaliers. Ces échanges ont vu le jour à l'aube du commerce international et ont persisté à travers les âges. Mais au cours des trente années de vie de ces accords de Bretton Woods, les opérations de change se limitaient à refléter les exigences réelles des entreprises et autres acteurs qui avaient besoin du change pour régler les factures et payer les marchandises. Lorsque les contrôles des changes ont été supprimés, le commerce de devises même, sous forme d'un marché où le change reflétait l'anticipation des fluctuations de prix, est devenu possible. Toutefois, les opérateurs n'avaient pas d'ordinateurs et il s'agissait alors pour eux de découvrir et de négocier ce marché qui restait «caché » dans l'enceinte d'un espace géographique.

Au tout début, une salle de marché était une salle dotée de bureaux, de lignes téléphoniques et d'une machine à calculer. Il pouvait également s'y trouver une cabine téléphonique centrale installée au milieu de la salle et qui servait à l'origine de lieu tranquille pour passer les appels internationaux qui, au début, devaient encore être demandés via la compagnie de téléphone ; seuls les appels nationaux pouvaient être passés directement. Le ticker (téléscripteur), qui débitait «50 mètres par jour» de cotations et titres

16. [NdT: il s'agit d'un dispositif de dialogues électroniques sur écran, ressemblant à un «webchat» sur internet, à travers lequel les traders communiquent des propositions de «deals », les commentent, les acceptent ou les rejettent]. 
d'actualité, comme l'a dit un ancien participant, était un dispositif essentiel. Les activités du parquet se concentraient sur une "recherche du marché », qui consistait à découvrir quel était le cours d'une devise et qui voulait l'échanger. Dans la citation suivante, un ancien responsable de négociation se souvient comment il courait continuellement après le marché :

P : (...) Donc il fallait constamment trouver quels étaient les cours dans chaque pays.

KK : Et vous le faisiez en appelant les banques ?

P : Oui. Et il y avait aussi des appels sur le télex provenant d'autres banques qui voulaient soit réaliser des opérations, soit savoir, simplement savoir où en était le dollar par rapport au franc suisse.

$\mathrm{KK}:(\ldots)$

P : Oui, on était courtiers pour des traders, chaque matin il fallait récupérer tous les cours d'Europe, Couronne danoise, Couronne suédoise, Couronne norvégienne, et autres, les devises nationales tous les matins, les taux d'ouverture. On les donnait aux traders, ils les calculaient en Francs suisses et les inscrivaient sur des grandes feuilles.

B : Et vous proposiez déjà le double cours, achat et vente ?

$\mathrm{P}$ : (...) Dans les banques suisses, les taux de change étaient déterminés par négociation, comme dans un bazar (etc.).

Si l'apprésentation est le transport de détails provenant d'emplacements géographiques et fuseaux horaires différents vers une zone d'activités particulière, il est sûr que des tentatives partielles d'apprésentation des marchés ont eu lieu avant l'introduction des écrans : les cours inscrits à la main sur les « grandes feuilles » dont parle P dans la citation ci-dessus étaient affichés sur des panneaux muraux et peuvent donc être considérés comme des premières tentatives d'apprésentation du marché. Lorsque les écrans sont apparus, ils n'étaient au début rien de plus que des remplaçants de ces «grandes feuilles»: des écrans sur lesquels les feuilles de cours manuscrites compilées par des secrétaires étaient projetées sur la base de photographies prises à partir des feuilles affichées dans la salle de marché. Cette forme d'apprésentation reposait sur une chaîne d'activités similaire à de nombreux égards à celle qui récupérait les cours à l'époque pré-écrans : il fallait cerner la position du marché en appelant ou envoyant des télex aux banques, écrire les réponses à la main (et peut-être recalculer les cours dans 
les devises nationales) et rendre ces informations accessibles en interne par une forme de présentation centrale.

Les écrans n'ont commencé à «apprésenter » plus directement une matrice d'intérêts épars et dissociés qu'en 1973, lorsque l'agence de presse britannique Reuters lança pour la première fois son système informatisé, Monitor, qui constitua la base d'un marché des changes électronique. Toutefois, Monitor n'apprésentait toujours que partiellement le marché, puisqu'il ne fournissait lui aussi que des cours indicatifs ${ }^{17}$. En revanche, il a tout de suite intégré des nouvelles textuelles (news). Les opérations proprement dites sont restées extérieures aux activités sur écran et ont été réalisées par téléphone et télex jusqu'en 1981, lorsque les services boursiers également développés par Reuters sont passés «en direct ${ }^{18}$ ». Mais, dès ses débuts, Monitor a changé radicalement l'un des aspects constitutifs de l'échange marchand : il répondait à la question « où est le marché ? ", c'està-dire qu'il indiquait quels étaient les cours des devises et qui pouvait être disposé à négocier.

Avant le marché sur écran, les cours différaient en fonction de l'emplacement et ils devaient être établis pour chaque nouvelle transaction par de longs et fastidieux processus d'appels téléphoniques, et de mises en attente pour les appels internationaux. Après l'introduction de Monitor, les cours sont soudainement devenus accessibles dans le monde entier à toute personne connectée au système. Avant le marché sur écran, il n'existait que des réseaux épars entre contreparties spécifiques. Après l'introduction des cotations informatisées sur écran, «le marché» ne résidait plus dans un réseau d'emplacements multiples, mais dans un seul, l'écran, qui pouvait être représenté à l'identique en tous lieux. Il avait acquis une présence et un profil propres, ainsi que ses propres propriétés temporelles. La conséquence économique de ce regroupement de tous les fragments de marché en un lieu unique a été la perte d'importance de l'arbitrage ${ }^{19}$. Les différences de prix entre emplacements rendues visibles à l'écran, même si elles ne concernent

17. [NdT: des cours de marchés sont dits «indicatifs» quant il s'agit simplement d'estimations approximatives ou de propositions de cours et non pas de cours fermes auxquels il est effectivement possible de traiter immédiatement].

18. READ, 1992, p. 283.

19. [NdT : l'arbitrage se base, dans ce contexte, sur la possibilité de profiter de l'écart de prix existant entre deux lieux de négociation pour une même devise]. 
que des cours indicatifs, seront rapidement éliminées, à mesure que les informations y afférentes deviendront accessibles à tous les traders connectés.

\section{LE MONDE DÉPLOYÉ DE L'ÉCRAN ET COMMENT LES TRADERS L'HABITENT}

Que cela signifie-t-il, pour la réalité de l'écran, d'être un «monde » et pour les traders d'habiter ce monde ? Nous pouvons prendre «l'inclusivité » des différentes apprésentations pour point de départ d'une réponse à cette question. La conséquence de ce qui a été dit dans la section précédente est que les programmes et circuits électroniques qui sous-tendent le marché sur écran ont rassemblé et implémenté sur un lieu unique les activités, temporalités et situations autrefois éparses des agents à l'œuvre: des courtiers et des comptables, des teneurs de marché (market makers) et des analystes, des ingénieurs financiers et des journalistes. Les activités de ces agents ont également servi à rassembler sur écran les états d'économies et de politiques nationales distantes et d'autres événements économiquement pertinents, ainsi que les archives du passé et les prédictions de l'avenir. C'est en ce sens que l'écran n'est pas simplement un «moyen » de transmission des messages et des informations. C'est un chantier de construction sur lequel est érigé tout un monde économique et épistémologique. Le caractère de «monde » de ce chantier apparait à travers les possibilités performatives des systèmes transactionnels implémentés à l'écran. Presque toutes les transactions institutionnelles en matière d'opérations de devises sont aujourd'hui réalisées sur écran; en outre, celui-ci peut être «parcouru » et de nouvelles fenêtres peuvent être ouvertes, ce qui donne l'impression d'une profondeur et d'une richesse d'expérience inépuisables.

Dans la littérature en sciences sociales, il n'est pas facile de trouver des critères permettant de déterminer le moment où nous devons compter quelque chose comme un «monde » à part entière. Nous pouvons toutefois nous appuyer sur la description phénoménologique de Schutz de la réalité quotidienne - sur sa structure de «monde de vie» (lifeworld) - pour inspecter l' "écran-monde» (screen world). Pour Schutz, l'expérience du monde de la vie quotidienne recouvre les caractéristiques suivantes : une tension spécifique de la conscience (un éveil attentif), une suspension du doute, une intentionnalité orientée vers le monde extérieur, une forme spécifique d'expérience de soi (celle du soi travaillant en tant que soi total), une forme spécifique de socialité (le monde intersubjectif commun de la 
communication et de l'action sociale) et une perspective temporelle spécifique qu'il a appelée «temps standard ${ }^{20}$ ». Schutz s'intéresse au monde du travail, et les caractéristiques qu'il énumère ont sans doute autant cours dans les échanges commerciaux sur écran que dans tout autre contexte de travail. Néanmoins, une élaboration spécifique de certaines de ces caractéristiques lorsqu'un monde de travail se déplace sur écran est possible. En d'autres termes, la caractérisation recherchée doit permettre à un habitat technologique de générer sa propre typification au sein d'une structure d'expérience globale. Nous suggérons que la plupart des caractéristiques de monde de Schutz sont élaborées spécifiquement dans les échanges marchands sur écran. Pour illustrer notre propos, nous nous concentrons sur la manière dont son éveil attentif, son intentionnalité et sa temporalité reflètent cette typification spécifique.

Lorsqu'il explique l'éveil attentif (wide-awakeness), Schutz décrit une «attention pleine et entière à la vie et à ses exigences », lorsque le soi travaillant «délimite le segment du monde qui est pragmatiquement pertinent » et que le contexte pertinent détermine la forme et le contenu de la pensée du sujet ${ }^{21}$. Dans ce contexte-ci, il est important de noter que les « exigences » du monde sur écran des opérateurs n'impliquent pas seulement une attention complète à la vie, mais une concentration et une élévation de l'expérience particulières, souvent décrites comme une forme d'activation et de stimulation bien supérieure au simple éveil attentif. Lorsque les traders arrivent le matin, ils s'installent sur leurs sièges, ils allument les écrans, et à partir de ce moment, leurs yeux seront collés aux écrans, leur regard capturé par l'écran, même quand ils se parlent ou crient, leur corps et le monde de l'écran fusionnant en ce qui semble être une immersion totale dans l'action à laquelle ils participent. Les traders commentent souvent l'intensité de ce genre d'expérience, comme dans le cas suivant :

Je pourrais être, je pourrais avoir dormi seulement trois heures la nuit précédente, être venu en train, complètement épuisé, j'arrive sur le parquet..., et, je ne bois pas de café non plus, juste du tonus, mon adrénaline crache immédiatement... C'est électrique ${ }^{22}$.

20. SCHUTZ, 1962, p. 229-30.

21. SCHUTZ, 1962, p. 212-14.

22. Cité dans LEVIN, 1999, p. 58 [NdT : cet extrait d'exemple se réfère au cas d'un parquet boursier]. 
Les traders peuvent également faire des commentaires sur l'atmosphère sociale accélérée qui résulte de tout ce qui se passe dans des laps de temps extrêmement courts, et sur la continuité de cette expérience : "On travaille, on se détend, on mange et on dort littéralement avec les marchés ${ }^{23}$. » Ce profond engagement semblerait laisser peu de place à la jouissance d'autres réalités non prépondérantes décrites par Schutz, parmi lesquelles il classe le monde des arts, l'expérience religieuse et les rêves ${ }^{24}$. Les habitants de ce monde-écran peuvent parvenir moins bien que d'autres à percer les murs de leur réalité et à déplacer l'accent sur une autre réalité ; même lorsqu'ils rêvent (comme nous l'a confié un trader), il est possible qu'ils rêvent du marché. Cette «adhésivité » du monde-écran peut également être liée à la composition économique des marchés respectifs. Comme l'a remarqué Abolafia, « la salle de marché n'est pas appréhendée comme un endroit où il suffit de se satisfaire, de traîner les pieds ou simplement survivre », comme dans d'autres cadres de travail, «c'est un endroit pour gagner ${ }^{25}$ ». Mais il se peut qu'une autre source de la tension accrue infligée à l'éveil attentif réside dans l'écran d'ordinateur lui-même et la nature temporelle de ses présentations du monde. Stone ${ }^{26}$ soutient à ce titre que le fait que la variété de canaux de signalisation soit moindre en régime de communication électronique qu'en interaction de face-à-face conduit à une situation où les facultés d'interprétation de la personne sont employées plus intensément. Peut-être un engagement plus intense, ouvert à des fantaisies interprétatives, se traduit-il non pas par une moindre qualité expérientielle des mondes en écran mais par une expérience «plus élevée » et une plus grande attraction.

Nous devons cependant souligner que nous ne prenons pas ici la réalité de l'écran en purs termes de matériels technologiques, systèmes de négociation ou logiciels. Notre intérêt porte en effet davantage sur l'écran en tant que surface textuellement élaborée, pleine de contenus, et qui encadre et présente un monde. Bien sûr, l'échafaudage microélectronique derrière la surface ne doit pas être ignoré, pas plus que ne doit l'être l'économie d'informations qui alimente les fenêtres de l'écran. Néanmoins, ce qui nous intéresse ici est que tous ces facteurs d'alimentation et de soutien convergent vers l'écran, où ils donnent collectivement naissance à ses caractéristiques de «monde ».

23. SCHWAGER, 1992, p. 60.

24. SCHUTZ, 1962, p. 231-59.

25. SCHUTZ, 1998, p. 10.

26. STONE, 1996, p. 36. 
Passons maintenant à une deuxième façon dont ces caractéristiques s'éloignent du monde schutzéen : la temporalité du monde en écran. Les problèmes temporels sont impliqués lorsque les traders évoquent la continuité et l'inévitabilité du marché sur écran, ou son caractère intersubjectif (thème que nous avons abordé ailleurs ${ }^{27}$ ). Il s'agit, en l'occurrence, de comprendre comment le temps est ancré dans la réalité de l'écran et constitue ce que nous appelons la nature de « déploiement et flux » (rollout-and-flow) de ce monde. Commençons par la notion de Schutz du monde de la vie quotidienne comme étant régi par le temps standard. Schutz a défini le temps standard comme l'intersection de ce qu'il a appelé le temps « cosmique» et le temps interne ou «durée». Ainsi, selon lui, nous percevons nos propres mouvements corporels comme des événements se produisant dans l'espace-temps, c'est-à-dire dans le temps homogène spatialisé qui est mesuré par les horloges et autres dispositifs et dans lequel les événements physiques se produisent. Mais, simultanément, nous percevons également ces mouvements en tant que changements dans une succession continue d'un courant de conscience interconnecté, celui que Schutz a appelé, à partir de Bergson, «durée ». La durée inclut l'expérience de vieillir ou de devenir, ainsi que le stock d'expériences évoluant continuellement grâce à l'émergence et la «sédimentation » d'une nouvelle expérience. La notion de durée est liée au phénomène indiqué par Bergson et Husserl lorsqu'ils affirment que la présence vécue perdure : le passé est incorporé dans le présent par les souvenirs et les reconstructions et il anticipe également l'avenir. Lorsque nous travaillons (Schutz pense aux actions physiques), nous appréhendons nos activités en tant qu'événements dans les temps interne et externe, unifiant ainsi les deux dimensions temporelles en un flux unique.

Tout ceci mériterait de plus amples précisions, mais nous n'analyserons ici qu'une caractéristique temporelle particulière du monde de l'écran, à savoir sa nature processuelle «brève » et insidieuse. Considérons d'abord que les notions de réalité et de monde ont tendance à être des notions spatiales. Historiquement, les marchés qui constituent actuellement les mondes de l'écran étaient des lieux de marché, des endroits physiques où acheteurs et vendeurs pouvaient se rencontrer et coordonner leurs intérêts. De même, nos concepts d'une réalité quotidienne, celui de Schutz y compris, sont généralement des concepts spatiaux. Nous voyons la réalité comme un 
environnement qui existe indépendamment de nous et dans lequel nous demeurons et accomplissons nos activités quotidiennes. Les notions même d' «habiter» et d'un «écran-monde» en écran telles qu'utilisées ici suggèrent également la spatialité ; elles suggèrent que l'idée d'un environnement spatial peut être élargie aux réalités électroniques puisqu'elles deviennent un «habitat» pour certains d'entre nous. Le problème avec ces notions, c'est qu'elles impliquent que le temps soit quelque chose qui passe dans cet habitat spatial, mais soit externe à l'habitat lui-même. Nous associons davantage l'existence d'un habitat à la matérialité physique d'un monde spatial qu'à une quelconque dimension temporelle. Nous exprimons également, souvent, la durabilité du monde physique comparé à l'espérance de vie humaine par des notions de monde spatialisantes. Mais la réalité de l'écran n'a pas cette durabilité. Elle ressemble plutôt à un tapis dont des petites sections sont déployées (rolled out) devant nous. Le tapis fonde l'expérience; nous pouvons marcher dessus, nous pouvons changer de position dessus. En même temps, nous devons imaginer le tapis se constituant à mesure qu'il est déployé. Ainsi, la réalité de l'écran (le tapis) est un processus, mais pas exactement à l'image d'une rivière qui coule mais au sens d'une masse d'eau identique se transférant d'un endroit à un autre. Elle est plutôt processuelle au sens d'une succession infinie de matière non identique se projetant vers l'avant à mesure qu'elle modifie l'écran. C'est ce que nous appelons le caractère de « déploiement et flux » de cette réalité.

Cette acception contraste avec des notions courantes de flux, que nous devrions examiner brièvement. On tend souvent à associer le flux directement soit avec les choses qui voyagent (par les réseaux), soit avec la fluidité. La première idée correspond aux mobilités croissantes de la vie contemporaine. Elle formule le phénomène selon lequel non seulement les personnes font des trajets, voyagent et migrent en nombres apparemment toujours croissants. Mais les messages et l'information se déplacent également. C'est tout particulièrement la circulation des communications qui soutient l'idée d'une société de réseaux en tant que société basée sur les flux d'informations ${ }^{28}$. Bien que cette idée soit importante, elle est restreinte à la transmission ou au transfert de choses qui restent somme toute identiques ; mais ce n'est pas ce à quoi nous pensons en évoquant un monde en écran constitué temporellement. La seconde notion, celle de fluidité, repose sur la 
distinction entre les liquides et les solides. Par exemple, les analystes qui mettent l'accent sur la fluidité conceptualisent l'état actuel de la modernité comme étant marqué par une transition de formes d'ordre et de tradition plus solides à des structures qui sont plus liquides et fluides, ou qui se dissolvent, comme dans la célèbre expression de Marx selon laquelle «tout ce qui est solide se dissout dans l' $^{\prime}$ air $^{29} »$. La libéralisation de l'éducation traditionnelle illustre cette tendance, de même que la dérégulation des marchés, la flexibilisation du travail et la décomposition et le remplacement des relations familiales traditionnelles ${ }^{30}$. Cette notion de «dissolution du solide» se rapproche davantage de notre propos, mais ce que nous voulons dire concernant la réalité de l'écran n'est pas qu'elle est nomade (sans itinéraire) et non marquée par les traces de la structure économique et sociale. Notre propos concerne la projection et la reconstitution de cette réalité en tant que réalité qui apparaît continuellement par bribes, comme l'environnement qui apparaît la nuit dans les phares d'une voiture et dont la portée est limitée à l'avant du véhicule. C'est cette brève durée de « résidence » dans le monde de l'habitant et son émergence par morceaux épisodiques que la notion de flux capture ici.

Nous suggérons également qu'il est possible de retenir des notions telles que celle de «monde » ou d' « habiter », tout en restant conscient de la courte vie et de l'évolution défilante de cette habitation particulière. L'écran qui déploie le monde dans lequel les traders se meuvent présente néanmoins un tel monde; il présente un environnement complexe composé de régions visitables et d'horizons qui fondent les activités. Le sol peut bouger continuellement et le monde de vie est celui d'un «vol habité ». Mais les traders sont capables de gérer ce flux; leurs façons $\mathrm{d}^{\text {' }}$ "habiter» sont adaptées à ce monde. Cette adaptation est parfaitement illustrée par la capacité des traders à suivre les fluctuations du marché pendant leurs opérations et en développant un «sixième sens » pour ces fluctuations. Les traders analysent également les tendances de fluctuation à court et long terme de leur monde en termes d'histoires et de «vues d'ensemble » qui leur donnent une certaine permanence pour un bref instant.

Nous souhaitons à présent évoquer brièvement une troisième manière dont les caractéristiques de monde schutzéennes sont reconfigurées dans le monde en écran. Pour Schutz et d'autres, l'intentionnalité est la notion qui capture le 
mieux notre orientation vers le monde extérieur en termes de projets et de plans. Nous assistons à la vie non seulement en tant qu'êtres «bien éveillés », mais nous y sommes dirigés par des motifs ("éveillés afin de »), par la volonté de provoquer des situations projetées que nous anticipons. Il s'agit à nouveau d'une assertion très générale correspondant à tous les mondes de travail. Le monde de l'écran est cependant un monde vers lequel les participants ne sont pas seulement orientés par des projets et des intentions, mais dans lequel ils sont positionnés. Pour pénétrer dans ce monde et en faire partie, un trader doit prendre une position sur le marché, ce qui signifie qu'il ou elle doit avoir acheté ou vendu des devises ou tout autre instrument financier.

En conséquence, les traders voient le marché à tout moment avec les yeux de quelqu'un qui y a des intérêts, des engagements et des investissements. Les corrélats immédiats de la nature «positionnée » de la participation au monde dans ce domaine sont les «risques» et les «vulnérabilités»: les opérateurs sont exposés aux risques des fluctuations du marché par le haut ou par le bas, en raison du fait qu'ils soient ou pas «à court» d'une devise sur laquelle ils risquent d'enregistrer des pertes. Le positionnement se traduit également par des émotions comme l'«avidité » et la «peur» (tous ces termes sont naturels) : la peur de perdre et une disposition au gain. Les pertes, en particulier, sont exprimées dans un vocabulaire qui retentit d'émotions de violence et d'attaque ressenties. Comme le formule un trader de la salle de marché de Zurich, les termes se rapportent «principalement au sexe et à la violence, et nombre d'entre eux semblent concerner la pénétration anale ». La liste que nous avons dressée comportait entre autres : « je me suis fait baiser ; je me suis fait exploser ; je me suis fait lessiver ; je me suis fait niquer ; je me suis fait enculer ; je me suis fait défoncer ; j'ai été massacré ».

C'est une description bien plus violente, émotionnelle et processuelle que celle proposée par Schutz. Elle introduit le corps en tant qu'objet de douleur ou d'épreuve physique, même si la source de la douleur n'est pas physique. L'intentionnalité avec laquelle nous avons commencé prend une toute autre saveur. Si le mode d'entrée dans ce monde (la seule façon d'être «sur le marché ») est de prendre une position, les intentions seront toujours élaborées sous la menace de cette épreuve potentielle et elles seront modelées par les conséquences de projets déjà accomplis. Si ceci est correct, les façons d'habiter dans ce monde des traders devraient en quelque sorte 
refléter l'expérience du positionnement et de l'exposition au risque. Et cela semble bien être le cas. L'attention que les traders portent au côté émotionnel de ce monde représente l'une des nuances intéressantes des marchés financiers en tant que formes de vie. Les traders parlent fréquemment et régulièrement de la nécessité de gérer les émotions, ils développent des méthodes de gestion de ces émotions et considèrent la gestion des émotions comme partie intégrante des compétences et du savoirfaire de l'activité boursière professionnelle ${ }^{31}$.

Nous avons ainsi défini les caractéristiques de l'écran comme monde en termes d'inclusivité, de nature temporelle et de «déploiement», et en termes d'intensité d'expérience positionnée des traders. Pour souligner le contraste avec la conception schutzéenne d'un monde, nous pouvons parler de flux « raccourci » de ce monde au lieu du temps standard expérimenté en son sein, d'attraction et de monopolisation au lieu d'éveil attentif et de réactivité et de structures de manque au lieu d'intentions ${ }^{32}$. Nous mettons une fois encore l'accent sur le fait que ces schémas concernent le média qui est habité. Clairement, si le monde en écran est un monde de flux, cela concerne les technologies, les systèmes de négociation et l'alimentation en contenu qui constituent ce monde et expliquent son évolution progressive. Les traders travaillant sur écran contribuent au flux par la durée de vie spécifique de leurs activités et par le texte qu'ils ajoutent, mais la durée de vie et les exigences d'information sont préalablement données par le monde en écran. L'élargissement et la réactivité résultent de l'étroitesse de la structure et de la «brièveté » temporelle du monde électronique, ainsi que de l'existence de conditions d'entrée. Nous soutenons que la réalité en réseaux des périodes antérieures où les marchés n'étaient pas incarnés sur écran n'a pas fait preuve de ces caractéristiques de temporalité. Nous pensons également qu'ils n'ont entraîné d'élargissements similaires qu'à certains moments, par exemple lorsque des marchés ont été connectés dans les transactions d'arbitrage.

\section{LA MATÉRIALITÉ DE L'ÉCRAN-MONDE : INFORMATION ET TRANSPARENCE}

L'utilisation de la notion de «monde » conduit nécessairement à la question du type de matérialité que peut avoir un système que nous appelons

31. Voir également : SCHWAGER, 1992 ; ABOLAFIA, 1996.

32. Voir KNORR CETINA, BRUEGGER, 2002b pour une élaboration de ce dernier point. 
«monde». Ce que cette section tente de faire, en conclusion, c'est d'explorer cette matérialité. En quoi consiste le «matériel » d'un système situé entièrement dans l'espace symbolique d'une réalité fournie électroniquement ? Et quel type de matérialité peut correspondre à un monde caractérisable en tant que flux ? La réponse la plus littérale à cette question est que cette matérialité réside dans le texte, dans la multiplicité des chiffres inscrits, des titres, des phrases et des messages que l'on trouve sur l'écran ; et c'est sur ce caractère textuel que nous voulons mettre l'accent. Il est en effet cohérent avec l'idée de flux, si nous appréhendons le texte comme en cours d'écriture et non en tant que produit fini. La notion d'écriture renvoie aux activités des agences d'information et des prestataires qui fournissent, outre les terminaux informatiques, les abonnements à l'écrit sur écran. Si nous concentrons l'analyse sur cet écrit, nous mettons en lumière toute une économie secondaire dont l'activité est de créer ce monde par des développements informatiques et d'écriture.

Mais nous pouvons également viser plus loin et tenter d'atteindre une compréhension qui prend en compte le contenu de l'écrit. D'abord, nous devons veiller à accorder suffisamment de poids au fait que les présentations textuelles centrales de l'écran ne sont pas fournies par les agences, mais par les traders ici considérés. Les traders «performent» le marché sur écran à partir de leurs deals électroniques, des prix ainsi cotés et des communications liées aux transactions. L'économie secondaire ne fournit que les moyens électroniques de ces interactions. Nous pouvons donc imaginer le contenu de l'écran divisé en représentations et éléments performatifs (la partie purement représentationnelle consistant, par exemple, en des évaluations d'événements et de situations économiquement pertinents). Ensuite, nous devons être conscients du fait que l'élément clé du segment représentationnel n'est pas la vérité au sens de faits durables dont la solidité provient d'une correspondance confirmée avec le monde, mais des nouvelles (news): des rapports incessants et presque instantanés d'événements et situations d'actualité. Lorsque nous avons utilisé ci-dessus le terme «apprésentation» au lieu de «représentation» pour évoquer le contenu de l'écran, nous l'avons fait pour indiquer la perte de la fonction représentationnelle impliquée par le passage de la vérité aux nouvelles ; en utilisant ce terme, nous avons également mis l'accent sur le rapprochement géographique et temporel réalisé. Un troisième point à noter est que non seulement les propositions de deals et les conversations sur écran, mais 
également les apprésentations d'événements et situations distants, ont le potentiel de stimuler les mouvements du marché.

Tout cela signifie que le contenu de l'écrit sur écran est constitué d'information. Une compréhension plus profonde de la matérialité du monde de l'écran révèle donc qu'il est informationnel. C'est le processus d'arrivée des informations qui « court-circuite » continuellement le flux de ce monde, modifiant l'image du monde et invitant les activités de négociation et d'interprétation à répondre à cette modification.

Nous avons illustré ailleurs avec plus de détail comment les différents contenus de l'écran et les opérations elles-mêmes constituaient des informations pour les traders ${ }^{33}$. Nous voulons ici ajouter un détail supplémentaire qui met également en lumière le caractère collectif du monde en écran. La matérialité de ce monde dépend d'un régime de transparence de l'information. En l'occurrence, la transparence fait avant tout allusion à la qualité de «vision au travers » du monde en écran. Elle implique la visibilité des activités et événement en cours par delà les zones géographiques au sein des communautés transactionnelles internes des banques d'investissement internationales et au sein des marchés spécialisés dans un instrument particulier (par exemple, dans le marché au comptant ou le marché d'options). Il convient d'apprécier l'étendue de cette visibilité. $\mathrm{Si}$, par exemple, au cours du processus de recherche d'un prix pour une option sur devises, le desk ${ }^{34}$ de trading de Zurich discute de l'option avec Londres, les autres desks d'options de la banque, distribués de par le monde entier, écoutent ces conversations :

KK : Ils écoutent (ces conversations) ? Ils peuvent (les voir)?

SJ : (...) Quand on discute de quelque chose avec Londres sur la ligne directe, tout le monde l'entend, ce qui signifie que tout le monde peut participer à la conversation. En gros, ils peuvent participer. Si vous avez des informations pertinentes que les deux autres qui sont en train de discuter n'ont pas, ces informations s'ajoutent (à la discussion) et (vous dites), hé, j'ai entendu parler de (ceci)... (etc.). (Responsable des opérations sur options)

33. KNORR CETINA, BRUEGGER, 2002a.

34. [NdT: unité fonctionnelle au sein de la salle de marché (ici, desk de "trading», par opposition, par exemple à desk de « vente »)]. 
Les traders pensent que certaines sortes d'informations peuvent être mieux évaluées localement grâce à des contacts, des réunions d'information, la lecture de la presse locale et des observations locales. Ce que le Ministère des Finances du Japon a en tête est plus facile à évaluer, pensent les traders, à Tokyo qu'à Zurich. Dans le cas étudié, ces observations ne se « sédimentent » pas dans le centre de négociation local pour participer de la connaissance implicite d'un endroit, ou de traders individuels. Elles sont également rendues accessibles à l'échelle mondiale grâce aux tableaux d'affichage internes (bulletin boards) des banques, un autre composant du régime de transparence décrit. Les tableaux d'affichage sont des forums électroniques partagés, sur lesquels les participants de divers centres de négociation postent de brèves observations confidentielles :

RD : Les informations les plus sensibles vont là-bas, les moins sensibles sont transmises au client ou aux contacts internes. En fait c'est le saint Graal !

L'avantage des tableaux d'affichage électroniques et de l'impulsion qu'ils donnent à la transparence, c'est qu'ils relient des centres de négociation distants grâce aux observations postées sur ce que ces centres voient localement, dans leur région du monde ${ }^{35}$. Mais cet avantage réside également dans le fait que les superviseurs, en suivant l'évolution des marchés sur ces tableaux, sont mis au courant des activités des traders et s'en trouvent en mesure de les juger. Sur un plan plus général, les superviseurs peuvent «assister» aux activités des traders en tant que membres de leur organisation, en même temps qu'ils suivent le marché, puisque lui-même se compose des activités des traders à l'échelle mondiale. Ils peuvent surveiller non seulement les cours faits, les cours indicatifs des autres banques, les informations et les analyses de celles-ci qui sont systématiquement affichées sur les écrans, mais également les conversations transactionnelles, les conversations informationnelles et les transactions effectuées par voie électronique.

Ce type de régime de transparence des informations soulève un certain nombre de questions ${ }^{36}$, mais nous ne pouvons ici nous concentrer que sur la

35. [NdT: les «centres de négociation » auxquels les auteurs se réfèrent ici sont les diverses salles de marché d'un même et unique établissement bancaire. Il s'agit ici de dispositifs de diffusion d'information «internes» (les bulletin boards électroniques), différents des dispositifs «de marché» étudiés plus haut (écrans de news et écrans de négociation électronique)].

36. Voir KNORR CETINA, BRUEGGER, 2001. 
façon dont il contribue à la matérialité du monde de l'écran et sur l'importance de ce régime eu égard à la situation des réseaux. D'abord, la notion de marché en tant que monde sur écran ne devient plausible que si ce monde contient suffisamment de «choses » (stuff) et si le volume et les couches d'informations, c'est-à-dire le matériel, sur l'écran sont proportionnels à la transparence, c'est-à-dire à ce que l'on peut voir à partir d'autres zones des nombreux réflecteurs de l'écran. Transparence équivaut également à visibilité, les traders servant de conduits de l'information. Les participants qui sont orientés vers et connaissent les «choses » de chacun, par le truchement de la transparence, connaitront également un certain niveau de coordination globale de la conscience qui leur permet de discriminer ce monde de la coordination plus binaire de la conscience dans les structures en réseau. Habiter l'écran n'est donc pas une aventure individuelle ou relationnelle, mais une aventure véritablement collective. Enfin, le fait que le monde en écran soit régi par un régime de transparence des informations a également des effets économiques directs. Les économistes abordent ce contraste en termes d'augmentation de l'efficience de ces marchés, par quoi ils entendent que les opportunités de profit excédentaires sont éliminées et que les prix reflètent davantage la meilleure information disponible ${ }^{37}$.

Traduit de l'anglais par WORDSHOP

37. Par exemple, BODIE, MERTON, 1995, p. 197. 
ABOLAFIA M. (1996), Making Markets: Opportunism and Restraint on Wall Street, Cambridge (Massachusetts), Harvard University Press.

ABOLAFIA M. (1998), "Markets as Cultures: An Ethnographic Approach", in Callon M. (ed.), The Laws of the Market, Oxford, Blackwell.

BAKER W. (1990), "Market Networks and Corporate Behavior”, American Journal of Sociology, vol. XCVI, p. 589-625.

BAKER W., FAULKNER R.R., FISHER G.A. (1998), "Hazards of the Market: The Continuity and Dissolution of Interorganizational Market Relationships", American Sociological Review, vol. LXIII, p. 147-177.

BANK FOR INTERNATIONAL SETTLEMENTS (1998), Central Bank Survey of Foreign Exchange and Derivatives Market Activity in April 1998: Preliminary Global Data, Bâle, BIS.

BAUMANN Z. (2000), Liquid Modernity, Cambridge, Polity Press.

BERMAN M. (1982), All That is Solid Melts Into Air: The Experience of Modernity, Londres, Verso.

BODIE Z., MERTON R.C. (1995), "The Informational Role of Asset Prices: The Case of Implied Volatility", in Crane D. et al. (eds.), The Global Financial System, Boston, Harvard Business School.

CASTELLS M. (1996), The Rise of the Network Society, New York, Harper \& Row.

DIMAGGIO P. (2001), «Introduction», in Dimaggio P. (ed.), The 21st Century, Princeton, Princeton University Press.

DIMAGGIO P., LOUCH H. (1998), "Socially Embedded Consumer Transactions: For What Sorts of Purchases do People Use Networks Most?, American Sociological Review, vol. LXIII, p. 619-37.

GERLACH M. (1992), Alliance Capitalism: The Social Organization of Japanese Business, Berkeley, University of California Press.

GRANOVETTER M. (1985), "Economic Action and Social Structure: The Problem of Embeddedness", American Journal of Sociology, vol. XCI, p. 481-510.

HAMILTON G., BIGGART N. (1993), "Market, Culture and Authority: A Comparative Analysis of Management and Organization in the Far East, in Granovetter M., Swedberg R. (eds.), The Sociology of Economic Life, Boulder (Colorado), Westview Press. 
KNORR CETINA K., BRUEGGER U. (2001), "Transparency Regimes and Management Content in Global Organizations. The Case of Institutional Currency Trading”, Journal of Knowledge Management, vol. V, p. 180-194.

KNORR CETINA K., BRUEGGER U. (2002a), "Global Microstructures: The Virtual Societies of Financial Markets”, American Journal of Sociology, vol. CVII, p. 905-950.

KNORR CETINA K., BRUEGGER U. (2002b), “Traders Engagement with Markets : A Postsocial Relationship”, Theory, Culture and Society, vol. XIX, p. 161-185.

LASCH C. (1978), The Culture of Narcissm, New York, W.W. Norton.

LAW J., HASSARD J. (eds.) (1999), Actor Network Theory and After, Oxford, Blackwell.

LEVIN P. (1999), "Gendering the Market: Temporality, Work and Gender on a National Futures Exchange", Communication, 94th Annual Meeting of the American Sociological Association, Chicago.

POWELL W.W., KOPUT K.W., SMITH-DOERR L. (1996), “Interorganizational Collaboration and the Locus of Innovation: Networks of Learning in Biotechnology", Administrative Science Quarterly, vol. XLI, p. 116-45.

READ D. (1992), The Power of News. The History of Reuters, Oxford, Oxford University Press.

SCHUTZ A. (1962), Collected Papers I, La Haye, Nijhoff.

SCHWAGER J.D. (1992), The New Market Wizards. Conversations with America's Top Traders, New York, Harper Collins.

STARK D. (1996), "Recombinant Property in East European Capitalism”, American Journal of Sociology, vol. CI, p. 993-1027.

STONE A.R. (1996), The War of Desire and Technology at the Close of the Mechanical Age, Boston, MIT Press.

UZZI B. (1999), "Embeddedness in the Making of Financial Capital: How Social Relations and Networks Benefit Firms Seeking Financing, American Sociological Review, vol. LXIV, p. 481-505. 